\title{
Análisis de los estudios clínicos sobre la eficacia de las técnicas alternativas al formocresol en las pulpotomías de dientes temporales
}

\author{
Calatayud J*, Casado I**, Álvarez C***
}

\section{RESUMEN}

Después de analizar los estudios clínicos de las técnicas de pulpotomía en dientes temporales alternativas al formocresol: hidróxido de calcio, electrocoagulación, glutaraldehido, sulfato férrico y Mineral Trióxido Agregado (MTA), hemos llegado a la conclusión de que las tres últimas son las mejores alternativas por presentar los mejores resultados y tener menor número de fracasos a lo largo del tiempo. En general todas tienden a empeorar sus resultados a medida que pasa el tiempo, desde un éxito clínico del $95-100 \%$ a los seis meses hasta un $80-90 \%$ a los tres años, excepto con MTA que en los pocos estudios clínicos existentes continúa manteniendo un resultado excelente (100\% a los dos años).

Palabras clave: Pulpotomía, Hidróxido de calcio, Electrocoagulación, Glutaraldehido Sulfato férrico, MTA.

\section{SUMMARY}

After analyzing clinical studies on alternatives to formocresol in primary tooth pulpotomies-calcium hydroxide, electrosurgery, glutaraldehyde, ferric sulphate and Mineral Trioxide Aggregate (MTA) the authors conclude that the last three are the most effective, as they present the best early results and the smallest number of failures over time. Generally speaking, the clinical success rate declines in all these techniques with time, from 95$100 \%$ after six months to $80-90 \%$ after three years. The only exception is MTA, which continues to exhibit excellent results (100\% success) after two years, according to the fairly small number of clinical studies published.

Key words: Pulpotomy, Calcium hydroxide, Electrosurgery, Glutaraldehyde, Ferric sulphate, MTA.

Aceptado para publicación: Noviembre 2005.

* Profesor Titular del Departamento de Profilaxis, Odontopediatría y Ortodoncia. Facultad de Odontología. Universidad Complutense de Madrid.

** Profesora Asociada. Departamento de Profilaxis, Odontopediatría y Ortodoncia. Facultad de Odontología. Universidad Complutense de Madrid.

*** Profesora de Materiales Odontológicos. Facultad de Ciencias de la Salud. Departamento de Odontología. Universidad Europea de Madrid.

Calatayud J, Casado I, Álvarez C. Análisis de los estudios clínicos sobre la eficacia de las técnicas alternativas al formocresol en las pulpotomías de dientes temporales. Av. Odontoestomatol 2006; 22 (4): 229-239. 


\section{INTRODUCCIÓN}

En 1904 John P. Buckley desarrolló el formocresol para su empleo en la terapéutica pulpar (1) y a partir de 1923 Charles A. Sweet lo utilizó para efectuar pulpotomías en dientes temporales (2), desde entonces su empleo se ha generalizado hasta el punto de ser en la actualidad la técnica más utilizada en todo el mundo (3), sin embargo, dados los posibles problemas de toxicidad del formocresol (3-5) otras técnicas se han venido desarrollando en los últimos años como alternativas a las pulpotomías con formocresol en dientes temporales.

En este trabajo pretendemos analizar todos los estudios clínicos, tanto los longitudinales como los ensayos clínicos, que hemos podido encontrar para evaluar la eficacia de las diferentes alternativas y la recomendación al profesional de cual o cuales reúnen las mejores opciones.

\section{MATERIAL Y MÉTODO}

Se han buscado los estudios a partir de PubMed, de la propia bibliografía de los trabajos consultados y de la base de datos Cochrane Revisiones Sistemáticas en el apartado "tratamiento pulpar para caries extensas en dentición temporal" revisada el uno de noviembre de 2002. De todos los estudios sólo hemos seleccionado los trabajos clínicos (con seres humanos), tanto los estudios longitudinales, los retrospectivos así como los ensayos clínicos.

Se valoró que los tratamientos fueran lo más similar entre si, en cada técnica, y se registraron los tiempos de control clínico y radiológico. Cuando los estudios tenían pacientes revisados en tiempos diferentes se obtuvo el promedio y deliberadamente establecimos cuatro tiempos de control: seis meses, un año, dos años y tres años; como algunos estudios no coincidían exactamente se ubicaron en los tiempos más próximos.

Los resultados se dieron en porcentaje de éxito tanto clínico como radiológico de forma independiente, dado que el éxito clínico es mayor que el radiológico en las técnicas de pulpotomía. Como el objetivo es mantener el diente temporal hasta su exfoliación con la erupción del sucesor permanente, sin que este último se afecte, el éxito clínico tiene prioridad (610) por ello se consideran éxitos clínicos siempre que no tengan signos clínicos agudos (dolor, fístula, movilidad excesiva...) ni cambios óseos de perdida de soporte importante (6-9). En algunos de los trabajos no se disponía de las dos variables clínica/radiológica y por ello sólo reflejamos la que existía.

También se valoraron otras variables:

1. La metamorfosis cálcica u obliteración de los conductos, un efecto secundario de muchos tratamientos de pulpotomía en dientes temporales pero que no se considera un fracaso radiológico (11-15). Sus datos se reflejan también en porcentaje.

2. Datos sobre afectación del sucesor permanente, tanto si se aceleraba como si se retrasaba su erupción, así como si erupcionaba con alguna hipoplasia o alteración del esmalte. Este tipo de información era poco frecuente en la mayoría de los estudios.

3. La aparición de reabsorción interna, especialmente en los casos de técnica con hidróxido de calcio, dado que es una de las causas de fracaso radiológico en los tratamientos de pulpotomía y especialmente con esa técnica.

\section{RESULTADOS}

Los resultados los vamos a exponer para cada una de las técnicas, tomándonos la libertad de hacer un pequeño recordatorio de las mismas para ayudar a centrar más cada uno de los métodos de pulpotomía.

\section{Hidróxido de calcio}

La técnica con hidróxido de calcio fue propuesta por primera vez por Hermann en 1930 (16) y es después de la técnica con formocresol la más antigua. Actualmente se sigue enseñando en los países nórdi$\cos (3)$.

En la técnica, tras la eliminación de la pulpa cameral, con cucharilla e instrumental rotatorio con mucha 
irrigación, se controla la hemorragia de los muñones del suelo de la cámara pulpar con una bolita de algodón durante 5-10 minutos. Tras limpiar de restos de sangre la cámara pulpar y observar que apenas sangran los muñones de los conductos radiculares se prepara una pasta de hidróxido de calcio, para ello se mezcla polvo de hidróxido de calcio proanálisis con agua estéril (17) o del grifo (18) o con suero salino estéril (18), la pasta se lleva al suelo de la cámara pulpar para cubrir los muñones de los conductos radiculares evitando que entre el muñón y la pasta de hidróxido de calcio se forme un coágulo sanguíneo (19). Los restos se eliminan de las paredes y se rellena el resto de la cámara pulpar con un cemento tipo IRM, óxido de zinc eugenol, oxifosfato de zinc...

En la tabla 1 se encuentran los resultados de 12 estudios clínicos que abarcan un tiempo de contro- les entre los seis meses a los dos años. En los resultados destaca como el porcentaje de éxito clínico y radiológico disminuye con el tiempo de una manera muy marcada. También se encuentran los datos sobre reabsorciones internas, dado que con esta técnica son muy frecuentes y suponen una de las principales causas de fracaso. Las reabsorciones internas tienden a aumentar a medida que pasa el tiempo. Hay que destacar que apenas hay datos sobre obliteración interna o metamorfosis cálcica ni sobre alteraciones sobre el diente permanente sucesor, posiblemente porque no se dan con esta técnica.

\section{Electrocoagulación}

Esta técnica también denominada electrofulguración o electrobisturí, se comenzó a utilizar en pulpotomías de dientes temporales en 1965 (29).

\section{TABLA 1.- PULPOTOMÍA DE HIDRÓXIDO DE CALCIO. RESULTADOS DEL ÉXITO CLÍNICO Y RADIOLÓGICO ASÍ COMO DE LOS CASOS DE REABSORCIONES INTERNAS REGISTRADOS EN 12 ESTUDIOS CLÍNICOS DESDE LOS 6 MESES A LOS 2 AÑOS TRAS EFECTUARSE EL TRATAMIENTO}

\begin{tabular}{|c|c|c|c|c|}
\hline \multirow[b]{2}{*}{ Tiempo } & \multirow[b]{2}{*}{ Autor/año } & \multicolumn{2}{|c|}{ Éxito } & \multirow{2}{*}{$\begin{array}{c}\text { Reabsorción } \\
\text { interna }\end{array}$} \\
\hline & & Clínico & Radiológico & \\
\hline \multirow[t]{3}{*}{6 meses } & Zander 1939(20) & - & $71 \%$ & - \\
\hline & Heilig 1984(21) & $100 \%$ & $88 \%$ & $6 \%$ \\
\hline & Promedio & $100 \%$ & $80 \%$ & $6 \%$ \\
\hline \multirow[t]{7}{*}{1 año } & Brown $1947^{(22)}$ & - & 90,2 & - \\
\hline & Shoemaker 1955(23) & $32 \%$ & - & - \\
\hline & Law 1956(24) & $46 \%$ & $46 \%$ & - \\
\hline & Doyle $1962^{(25)}$ & $71,4 \%$ & $42,8 \%$ & $62,5 \%$ \\
\hline & Schröder 1978(19) & $67 \%$ & - & $33,3 \%$ \\
\hline & Gruythuysen $1997^{(18)}$ & 87,7 & - & - \\
\hline & Promedio & $60,8 \%$ & $59,6 \%$ & $47,9 \%$ \\
\hline \multirow[t]{7}{*}{2 años } & Via 1955((17) & $31,1 \%$ & - & $69 \%$ \\
\hline & Morgan 1960(26) & $90 \%$ & - & - \\
\hline & Magnusson $1970^{(27)}$ & - & $24 \%$ & $66,9 \%$ \\
\hline & Schröder $1978^{(19)}$ & $59 \%$ & - & - \\
\hline & Gruytuysen 1997(18) & $80,4 \%$ & - & $5,5 \%$ \\
\hline & Waterhouse $2000^{(28)}$ & $77 \%$ & $77 \%$ & $6 \%$ \\
\hline & Promedio & $67,5 \%$ & $50,5 \%$ & $36,8 \%$ \\
\hline
\end{tabular}


La técnica sigue la pauta ya comentada, así tras la eliminación de la pulpa cameral, con cucharilla e instrumental rotatorio con mucha irrigación, se controla la hemorragia de los muñones del suelo de la cámara pulpar con una bolita de algodón durante 510 minutos. Tras limpiar de restos de sangre la cámara pulpar y observar que apenas sangran los muñones de los conductos radiculares se aplica una descarga de corriente eléctrica, durante 1 ó 2 segundos, con el electrodo colocado a una distancia de 12 milímetros del muñón amputado y repitiendo la operación en todos los muños a intervalos de 5-10 segundos para evitar el excesivo calentamiento de la cámara pulpar. La maniobra se repite $2-3$ veces por cada muñón, colocando en cada intervalo una bolita de algodón para que absorba cualquier resto de sangre o fluidos antes de aplicar la corriente eléctrica $(32,33)$. Los electrobisturís que se han empleado hasta la fecha son el Hyfrecator 705A (31-33) y el Storbex Ultron (30), siempre a media potencia. Finalmente se rellena la cámara pulpar con un cemento tipo IRM, oxido de zinc eugenol, oxifosfato de zinc, como en el caso anterior.

En la tabla 2 se encuentran los resultados de cuatro estudios clínicos que abarcan un tiempo de controles entre los seis meses a los dos años. Los resultados son sorprendentes puesto que en los estudios con controles a más largo plazo los resultados clínicos y radiológicos mejoran en contra de la lógica de man- tenerse o disminuir. La obliteración de conductos sólo aparece en uno de los estudios a seis meses y no hay datos de alteración en el sucesor permanente.

\section{Glutaraldehido}

El glutaraldehido o aldehido glutárico se introdujo en pulpotomías de dientes temporales en 1973 (34). Este compuesto se emplea por su capacidad para fijar los tejidos así como por su poder antiséptico. Hay dos tipos de glutaraldehido (35): 1) el alcalino o tamponado, que es más potente y que una vez activado tiene una duración de 15-30 días; y 2) el ácido que tiene una vida inicialmente ilimitada. En la práctica se emplea al $2 \%$ puesto que no se han encontrado diferencias al utilizarlo al $2 \%$ o al $5 \%$, ni en la forma alcalina o ácida (36); además, al $2 \%$ es más manejable y menos irritante (37).

La técnica es muy parecida a la empleada con formocresol. Tras la eliminación de la pulpa cameral, con cucharilla e instrumental rotatorio con mucha irrigación, se controla la hemorragia de los muñones del suelo de la cámara pulpar con una bolita de algodón durante 5-10 minutos. Tras limpiar de restos de sangre la cámara pulpar y observar que apenas sangran los muñones de los conductos radiculares se coloca en la cámara pulpar una bolita de algodón impregnada en glutaraldehido al $2 \%$ durante cinco

\section{TABLA 2.- PULPOTOMÍA POR ELECTROCOAGULACIÓN. RESULTADOS DEL ÉXITO CLÍNICO Y RADIOLÓGICO ASÍ COMO DE LOS CASOS DE OBLITERACIÓN DE CONDUCTOS REGISTRADOS EN 4 ESTUDIOS CLÍNICOS DESDE LOS 6 MESES A LOS 2 AÑOS TRAS EFECTUARSE EL TRATAMIENTO}

\begin{tabular}{|lcccc|}
\hline & & \multicolumn{2}{c}{ Éxito } & Obliteración \\
Tiempo & Autor/año & Clínico & Radiológico & conductos \\
\hline \multirow{2}{*}{6 meses } & López Nicolás 1993(30) & $97,3 \%$ & $94,6 \%$ & - \\
& Fishman 1996(31) & $77,3 \%$ & $54,5 \%$ & $9 \%$ \\
\hline 1 año & Promedio & $\mathbf{8 7 , 3 \%}$ & $74,5 \%$ & - \\
\hline 2 años & Dean 2002(32) & $96 \%$ & $84 \%$ & - \\
\hline
\end{tabular}


minutos (36-42). Finalmente se rellena la cámara pulpar con un cemento tipo IRM, oxido de zinc eugenol, oxifosfato de zinc, como en los casos anteriores.

En la tabla 3 se encuentran los resultados de 10 estudios clínicos que abarcan un tiempo de controles entre los seis meses a los tres años. En los resultados destaca como el porcentaje de éxito clínico y radiológico tiende a disminuir con el tiempo pero aun así alcanza unos resultados bastante buenos. Por otra parte la metamorfosis cálcica también tiende a aumentar con el tiempo. Sobre el sucesor permanente encontramos que la erupción se encuentra adelantada en el $15 \%$ de los casos (39) y estos no presentan defectos del esmalte (46).

\section{Sulfato férrico}

El sulfato férrico se ha utilizado al 15,5\% (Astringedent ${ }^{\circledR}$ ) y actualmente al $20 \%$ (Viscostat ${ }^{\circledR}$ ). Este compuesto de hierro se utiliza por su acción fuertemente hemostática y su efecto bactericida moderado (12), pero no tiene acción fijadora de tejidos o momificante $(10,12)$.

La técnica sigue la pauta ya comentada, así tras la eliminación de la pulpa cameral, con cucharilla e instrumental rotatorio con mucha irrigación, se controla la hemorragia de los muñones del suelo de la cámara pulpar con una bolita de algodón durante 510 minutos. Tras limpiar de restos de sangre la cámara pulpar y observar que apenas sangran los muñones se aplica el Viscostat $\AA$, en unas jeringas especiales y con unas boquillas metálicas con punta de cepillo (dentoinfusor), apretando sobre cada uno de los muñones radiculares del suelo de la cámara pulpar, presionado sobre cada uno unos 10-15 segundos. Posteriormente se limpia con el chorro de agua y se seca quedando la cámara de color amarillo-marrón. Si tras la aplicación sangra algún muñón se puede volver a aplicar (12). Como en todas las

\begin{tabular}{|c|c|c|c|c|c|}
\hline \multicolumn{6}{|c|}{$\begin{array}{l}\text { TABLA 3.- PULPOTOMÍA CON GLUTARALDEHIDO. RESULTADOS DEL ÉXITO CLÍNICO } \\
\text { Y RADIOLÓGICO ASÍ COMO DE LOS CASOS DE OBLITERACIÓN DE CONDUCTOS } \\
\text { REGISTRADOS EN } 10 \text { ESTUDIOS CLÍNICOS DESDE LOS } 6 \text { MESES A LOS } 3 \text { AÑOS TRAS } \\
\text { EFECTUARSE EL TRATAMIENTO }\end{array}$} \\
\hline Tiempo & Autor/año & Clínico & $\begin{array}{l}\text { ito } \\
\text { Radiológico }\end{array}$ & $\begin{array}{c}\text { Obliteracón } \\
\text { de conductos }\end{array}$ & $\begin{array}{c}\text { Reabsorción } \\
\text { interna }\end{array}$ \\
\hline 6 meses & $\begin{array}{l}\text { Kopel } 1980^{(37)} \\
\text { Fuks } 1986^{(38)} \\
\text { Prakash } 1989^{(42)} \\
\quad \text { Promedio }\end{array}$ & $\begin{array}{l}91,6 \% \\
94,3 \% \\
100 \% \\
95,3 \%\end{array}$ & $\begin{array}{l}75 \% \\
- \\
100 \% \\
87,5 \%\end{array}$ & $\begin{array}{l}- \\
- \\
-\end{array}$ & $\begin{array}{l}- \\
-\end{array}$ \\
\hline 1 año & $\begin{array}{c}\text { García Godoy 1983(43) } \\
\text { Fuks 1986(38) } \\
\text { Alacam 1989(40) } \\
\text { Shumayrikh 1999(44) } \\
\text { Fernández 2000(41) } \\
\text { Promedio }\end{array}$ & $\begin{array}{c}96,4 \% \\
90,4 \% \\
96 \% \\
96,5 \% \\
100 \% \\
95,8 \%\end{array}$ & $\begin{array}{c}96,4 \% \\
- \\
92 \% \\
75,8 \% \\
100 \% \\
91 \%\end{array}$ & $\begin{array}{l}- \\
7,7 \% \\
- \\
- \\
- \\
-\end{array}$ & $\begin{array}{l}- \\
7,5 \% \\
7 \% \\
- \\
7.2 \%\end{array}$ \\
\hline 2 años & $\begin{array}{l}\text { Tsai } 1993^{(36)} \\
\text { Fuks } 1990^{(39)} \\
\text { Promedio }\end{array}$ & $\begin{array}{l}98 \% \\
82 \% \\
90 \%\end{array}$ & $\begin{array}{c}78,7 \% \\
- \\
78,7 \%\end{array}$ & $\begin{array}{l}18 \% \\
40 \% \\
29 \%\end{array}$ & $\begin{array}{l}- \\
-\end{array}$ \\
\hline 3 años & García Godoy 1986(45) & $94,1 \%$ & - & - & - \\
\hline
\end{tabular}


pulpotomías se rellena la cámara con cemento (IRM, OZE, fosfato de zinc...)

En la tabla 4 se encuentran los resultados de seis estudios clínicos que abarcan un tiempo de controles entre los seis meses a los tres años. En los resultados destaca como el porcentaje de éxito clínico y radiológico tiende a disminuir con el tiempo pero aun así alcanza unos resultados bastante buenos. Por otra parte la metamorfosis cálcica también tiende a aumentar con el tiempo. Además, no hay alteraciones en el esmalte del diente permanente sucesor (9) y en cerca del $10 \%$ de los casos está adelantada su erupción $(8,9)$.

\section{Mineral Trióxido Agregado (MTA)}

El MTA es un cemento muy prometedor en el campo de la endodoncia. Fue autorizado por la Food and Drug Administration (Departamento del Ministerio de Sanidad de los Estados Unidos) en 1998 (48) y comenzó a utilizarse en pulpotomías de dientes temporales en 2001 (13). El preparado que se emplea es el gris dado que el blanco tiene peores resultados (48).

La técnica es como hemos descrito en los casos anteriores y una vez que se ha eliminado la pulpa cameral y controlado la hemorragia se prepara el cemento MTA. El polvo gris de MTA se mezcla con suero salino en una proporción de tres partes de polvo por una de líquido. Una vez mezclado se lleva a la cámara pulpar con un portaamalgamas y se adapta al suelo con la presión de una bolita de algodón húmeda. Los restos se eliminan de las paredes y se rellena el resto de la cámara pulpar con un cemento tipo IRM, oxido de zinc eugenol, oxifosfato de zinc, como en los casos anteriores.

\begin{tabular}{|c|c|c|c|c|}
\hline \multicolumn{5}{|c|}{$\begin{array}{c}\text { TABLA 4.- PULPOTOMÍA CON SULFATO FÉRRICO. RESULTADOS DEL ÉXITO CLÍNICO } \\
\text { Y RADIOLÓGICO ASÍ COMO DE LOS CASOS DE OBLITERACIÓN DE CONDUCTOS } \\
\text { REGISTRADOS EN } 6 \text { ESTUDIOS CLIINICOS DESDE LOS } 6 \text { MESES A LOS } 3 \text { AÑOS TRAS } \\
\text { EFECTUARSE EL TRATAMIENTO }\end{array}$} \\
\hline \multirow[b]{2}{*}{ Tiempo } & \multirow[b]{2}{*}{ Autor/año } & \multicolumn{2}{|c|}{ Éxito } & \multirow{2}{*}{$\begin{array}{l}\text { Obliteración } \\
\text { de conductos }\end{array}$} \\
\hline & & Clínico & Radiológico & \\
\hline \multirow[t]{3}{*}{6 meses } & Fei $1991^{(12)}$ & $100 \%$ & $96,3 \%$ & - \\
\hline & Smith $2000^{(9)}$ & $99 \%$ & $80 \%$ & - \\
\hline & Promedio & $99,5 \%$ & $88,2 \%$ & - \\
\hline \multirow[t]{3}{*}{1 año } & Fei $1991^{(12)}$ & $100 \%$ & $96,3 \%$ & $48,3 \%$ \\
\hline & Smith $2000^{(9)}$ & $98 \%$ & $74 \%$ & $19,6 \%$ \\
\hline & Promedio & $99 \%$ & $85,2 \%$ & $33,9 \%$ \\
\hline \multirow[t]{5}{*}{2 años } & Fuks $1997^{(8)}$ & $92,7 \%$ & - & $18,2 \%$ \\
\hline & Smith $2000^{(9)}$ & $93 \%$ & $81 \%$ & $22,8 \%$ \\
\hline & Ibricenic $2000^{(47)}$ & $100 \%$ & $97,2 \%$ & - \\
\hline & Casas $2003^{(10)}$ & $94,5 \%$ & $61 \%$ & $71 \%$ \\
\hline & Promedio & $95 \%$ & $79,7 \%$ & $37,3 \%$ \\
\hline \multirow[t]{3}{*}{3 años } & Smith $2000^{(9)}$ & $81 \%$ & $74 \%$ & $33,3 \%$ \\
\hline & Casas $2004^{(48)}$ & - & $67 \%$ & $60 \%$ \\
\hline & Promedio & $81 \%$ & $70 \%$ & $46,2 \%$ \\
\hline
\end{tabular}


En al tabla 5 se encuentran los resultados de seis estudios clínicos que abarcan un tiempo de controles entre los seis meses a los tres años (un séptimo estudio presentó resultados preliminares) $(50,51)$. Los resultados son excelentes tanto clínicos como radiológicos puesto que tienen éxito el $100 \%$ de los casos y no hay disminución con el tiempo. También se observa que con el paso del tiempo si tiende a aumentar las obliteraciones de los conductos radiculares. No hay datos de alteración en los dientes sucesores permanentes.

\section{DISCUSIÓN}

En este trabajo no se ha efectuado una comparación con metaanálisis porque una metodología rigurosa obligaría a rechazar muchos de los trabajos consultados; además, este estudio no tiene como objetivo establecer una comparación con una hipótesis de que tratamiento es mejor. El objetivo principal es dar una información sobre la eficacia estimada así como de la evolución de cada una de las alternativas analizadas dado que esto puede ser de un gran interés para el clínico.
Se puede observar fácilmente que la técnica de pulpotomía con hidróxido de calcio presenta unos resultados más pobres que el resto. Para algunos autores los resultados pueden mejorase evitando que se forme el coágulo de sangre sobre el muñón que impide el contacto directo del hidróxido de calcio sobre dicho muñón, sin embargo, esto no siempre es posible a pesar de ser muy cuidadoso (25). También podemos ver como los estudios más antiguos presentan resultados más bajos que los más modernos. Esto se debe a que en los primeros tiempos se separaba la base de hidróxido de calcio (alcalina) del suelo de la cámara pulpar del cemento de relleno (oxifosfato de zinc, ácido) con parafina (19) o asbestos (21) evitándose un buen sellado, que es básico para el éxito. Aun corrigiendo este factor podemos observar que los resultados, incluso de estos trabajos más recientes, son más bajos que con las otras técnicas.

La técnica con electrocoagulación da resultados contraintuitivos, con un aumento paradójico de resultados a medida que los estudios hacen las evaluaciones a más largo tiempo. Esta aparente incongruencia puede estar motivada por el escaso número de trabajos, sólo cuatro, y tal vez por ello la técnica no se

\section{TABLA 5.- PULPOTOMÍA CON MTA. RESULTADOS DEL ÉXITO CLÍNICO Y RADIOLÓGICO ASÍ COMO DE LOS CASOS DE OBLITERACIÓN DE CONDUCTOS REGISTRADOS EN 6 ESTUDIOS CLÍNICOS DESDE LOS 6 MESES A LOS 3 AÑOS TRAS EFECTUARSE EL TRATAMIENTO}

\begin{tabular}{|lcccc|}
\hline \multirow{2}{*}{ Tiempo } & Autor/año & Clínico & $\begin{array}{c}\text { Éxito } \\
\text { Radiológico }\end{array}$ & $\begin{array}{c}\text { Obliteración } \\
\text { de conductos }\end{array}$ \\
\hline \multirow{2}{*}{6 meses } & Agamy 2004(49) & $100 \%$ & $100 \%$ & - \\
& Maroto 2004-2005(50,51)) & $100 \%$ & $100 \%$ & $56,5 \%$ \\
& Naik 2005(52) & $100 \%$ & $100 \%$ & $60 \%$ \\
& Promedio & $100 \%$ & $100 \%$ & $58,3 \%$ \\
\hline 1 año & Eidelman 2001(13) & $100 \%$ & $100 \%$ & $41 \%$ \\
& Agamy 2004(49) & $100 \%$ & $100 \%$ & $58 \%$ \\
& Maroto 2004(50) & $100 \%$ & $100 \%$ & $64 \%$ \\
\hline 2 años & Promedio & $100 \%$ & $100 \%$ & $7,9 \%$ \\
\hline 3 años & Farsi 2005 & $15 \%$ & $100 \%$ & $58 \%$ \\
\hline
\end{tabular}


ha generalizado. En cualquier caso se necesitan más trabajos sobre esta técnica para concretar los factores que influyen en su éxito de una forma predecible.

A nuestro juicio estas dos técnicas deberían de evitarse por el clínico, la primera porque otras tienen mejores resultados y la segunda por lo arbitrario de los mismos y la falta de más estudios que aclaren sus resultados paradójicos.

Las técnicas con glutaraldehido, sulfato férrico y MTA son, a nuestro juicio, las que podemos proponer como alternativas, pero tenemos que llamar la atención a algunos aspectos.

La pulpotomía con glutaraldehido tampoco está exenta del problema de la toxicidad atribuido al formocresol, aunque más reducido, sobre todo porque se emplea al $2 \%$ dado que cuando el formocresol se utiliza diluido al 1:5 las diferencias con el glutaraldehido se reducen considerablemente (54-56). Por otra parte, si se emplea la forma alcalina de glutaraldehido tiene que estar fresca, recién hecha $(34,37,38)$, ya que tiene una vida de 15-30 días.
La técnica con sulfato férrico tiene la ventaja de carecer de la toxicidad del formocresol y del glutaraldehido, además de ser muy rápida de aplicación (no hay que esperar cinco minutos) y fácil. Es una técnica interesante aunque sería deseable el efectuar más estudios (tiene seis estudios clínicos) y a más largo plazo, puesto que con el paso del tiempo los resultados parecen resentirse más que con otras técnicas.

La pulpotomía con MTA es la que hasta la fecha aporta los mejores resultados tanto clínicos como radiológicos (sobre un 100\%) pero presenta algunos inconvenientes. El primero es el alto coste de su producto comercial ProRoot ${ }^{\circledR}$. El segundo problema es que hay pocos estudios a dos, tres y más años, aunque muy posiblemente en los próximos años asistamos a la aparición de más. En la actualidad hay pocos estudios con MTA (seis estudios clínicos), pero tienen la ventaja de que los resultados de todos son muy coincidentes.

Como resumen queremos presentar en la tabla 6 una especie de síntesis con los datos más reseñables de las tres técnicas que a nuestro juicio se pue-

\begin{tabular}{|c|c|c|c|c|}
\hline $\begin{array}{l}\text { TABLA 6.- DATOS RE } \\
\text { LOS } 6 \text { MESES A LO } \\
\text { FORMOCRESOL. TAMBI } \\
\text { NÚMERO DE ESTUDIOS }\end{array}$ & $\begin{array}{l}\text { YDEADOS DI } \\
\text { AÑOS DE LA } \\
\text { SE INCLUYEN } \\
\text { YICOS Y CAS }\end{array}$ & $\begin{array}{l}\text { XITOS CLÍNIC } \\
\text { ICAS DE PUL } \\
\text { E INTRODUCC } \\
\text { BLITERACIÓN }\end{array}$ & $\begin{array}{l}\text { ADIOLOGICO } \\
\text { ÍA ALTERNAT } \\
\text { LA TÉCNICA } \\
\text { NDUCTOS RA }\end{array}$ & $\begin{array}{l}\text { SDE } \\
\text { AL } \\
\text { ÍNICA, } \\
\text { LARES }\end{array}$ \\
\hline & & Glutaraldehido & Sulfato férrico & MTA \\
\hline Año del primer estudio clín & & 1973 & 1991 & 2001 \\
\hline Núm. de estudios clínicos & & 10 & 6 & 6 \\
\hline 6 meses & Clínico & $95 \%$ & $100 \%$ & $100 \%$ \\
\hline & Radiológico & $90 \%$ & $90 \%$ & $100 \%$ \\
\hline 1 año & Clínico & $95 \%$ & $100 \%$ & $100 \%$ \\
\hline & Radiológico & $90 \%$ & $85 \%$ & $100 \%$ \\
\hline 2 años & Clínico & $90 \%$ & $95 \%$ & $100 \%$ \\
\hline & Radiológico & $80 \%$ & $80 \%$ & $100 \%$ \\
\hline 3 años & Clínico & $90 \%$ & $80 \%$ & $95 \%$ \\
\hline & Radiológico & - & $70 \%$ & - \\
\hline Obliteración de conductos & 1 año & $10 \%$ & $35 \%$ & $65 \%$ \\
\hline & 2 años & $30 \%$ & $45 \%$ & - \\
\hline
\end{tabular}


den emplear como alternativas a la pulpotomía con formocresol, destacando la estimación de éxitos clínicos y radiológicos (con datos redondeados y adaptados) para que el clínico tenga una orientación de lo que puede esperar al aplicar estas técnicas.

\section{BIBLIOGRAFÍA}

1. Buckley JP. A rational treatment for putrescent pulps. Dent Rev 1904;18:1193-7.

2. Sweet CA. Root canal treatment in deciduous teeth, incluiding pulp exposure. Pacific Dent Gaz 1923;31:718-21.

3. Avram DC, Pulver F. Pulpotomy medicaments for vital primary teeth. Surveys to determine and attitudes in pediatric dental practice and in dental school throught the world. J Dent Child 1989; 56(6):426-34.

4. Ketley CE, Goodman JR. Formocresol toxicity: is there a suitable alternative for pulpotomy of primary molars?. Int J Paediatr Dent 1991; 2(2): 67-72.

5. Lewis B. Formaldehyde in dentistry: a review of the millenium. J Clin Pediatr Dent 1998;22(2): 167-77.

6. Magnusson BO. Therapeutic pulpotomies in primary molars with the formocresol technique. A clinical and histological follow up. Acta Odontol Scand 1978;36(3):157-65.

7. Hicks MJ, Barr ES, Flaitz CM. Formocresol pulpotomies in primary molars: a radiographic study in a pediatric dentistry practice. J Pedod 1986;10(4):331-9.

8. Fuks AB, Holan G, Davis JM, Eidelman E. Ferric sulfate versus dilute formocresol in pulpotomized primary molars: a long-term follow up. Pediatr Dent 1997;19(5):327-30.

9. Smith NL, Seale NS, Nunn ME. Ferric sulfate pulpotomy in primary molars: a retrospective study. Pediatr Dent 2000;22(3):192-9.
10. Casas MJ, Layng MA, Kenny DJ, Johnson DH, Judd PL. Two-year outcomes of primary molar ferric sulfate pulpotomy and root canal therapy. Pediatr Dent 2003; 25(2): 97-102.

11. Flaitz CM, Barr ES, Hicks MJ. Radiographic evaluation of pulpal therapy of primary anterior teeth. J Dent Child 1989;56(3):182-5.

12. Fei A-L, Udin RD, Johnson R. A clinical study of ferric sulfate as a pulpotomy agent in primary teeth. Pediatr Dent 1991;13(6): 327-32.

13. Eidelman E, Holan G, Fuks AB. Mineral trioxide aggregate vs formocresol in pulpotomized primary molars: a preliminary report. Pediatr Dent 2001;23(1):15-8.

14. Holan G, Fuks AB, Keltz N. Success rates of formocresol pulpotomy in primary molars restored with stainles steel crown vs amalgam. Pediatr Dent 2002;24(3):212-6.

15. Farsi N, Alamondi N, Balto K, Mushayt A. Success of mineral trioxide aggregate in pulpotomized primary molars. J Clin Pediatr Dent 2005;29(4): 307-11.

16. Hermann. Dentinobliteration der Wurzelkanäle nach Behandlung mit Calcium. Zahnärztliche Rundschau 1930;39(21):888-99.

17. Via WF Jr. Evaluation of deciduous molars trated by pulpotomy and calcium hydroxide. JADA 1955; 50(1):34-43.

18. Gruythuysen RJM, Weerheijm KL. Calcium hydroxide pulpotomy with a light-cured cavityseding materials after two years. J Dent Child 1997;64(4):251-3.

19. Schröder U. A 2-year follow-up of primary molars, pulpotomized with a gentle technique and capped with calcium hydroxide. Scand J Dent Res 1978; 86(4);273-8.

20. Zander HA. Reaction of the pulp to calcium hydroxide. J Dent Res 1939; 18: 373-9. 
21. Heiling J, Yates J, Siskin M, Mcknight J, Turner J. Calcium hydroxide pulpotomy for primary teeth: a clinical study. JADA 1984;108(5):775-8.

22. Brown WE Jr. The pulpotomy technic for the management of vital exposed pulps in primary and young permanent teeth. Alumni Bull Univ Mich Sch Dent 1947;48:14-6.

23. Shoemaker CP. Results of tweenty-eight pulpotomies. JADA 1955;50(1):71.

24. Law DB. An evaluation of vital pulpotomy technique. J Dent Child 1956;23(1):40-4.

25. Doyle WA, McDonald RE, Mitchell DF. Formocresol versus calcium hydroxide in pulpotomy. $\mathrm{J}$ Dent Child 1962;29(2):86-97.

26. Morgan ML. A clinical and roentgenographic evaluation of the effectiveness of calcium hydroxide in vital pulp therapy. J Dent Child 1960; 27(3): 243.

27. Magnusson B. Therapeutic pulpotomy in primary molars- clinical and histological follow-up I. Calcium hydroxide paste as wound dressing. Odontol Rev 1970;21(4):415-31.

28. Waterhouse PJ, Nunn JH, Whitworth JM. An investigation of the relative efficacy of Buckley's formocresol and calcium hydroxide in primary molar vital pulp therapy. Br Dent J 2000; 188(1): 32-6.

29. Auderman I. Indications for use of electrosurgery in pedodontics. Symposium on electrosurgery. Dent Clin N Am 1982;26(4):711-28.

30. López-Nicolás M, García C, Cabrerizo M, Romero A. Pulpotomía con electrobisturí alternativa a la pulpotomía clásica. Odontol Pediatr 1993;2(1): 9-13.

31. Fishman SA, Udin RD, Good DL, Rodef F. Success of electrofulguration pulpotomies covered by zinc oxide and eugenol or calcium hydroxide: a clinical study. Pediatr Dent 1996; 18(5): 385-90.
32. Dean JA, Mack RB, Fulkerson BT, Sanders BJ. Comparision of electrosurgical and formocresol pulpotomy procedures in children. Int J Paediatr Dent 2002;12(3):177-82.

33. Mack RB, Dean JA. Electrosurgical pulpotomy: a retrospective human study. J Dent Child 1993; 60(2):107-14.

34. Wemes JC, S'Gravenmade EJ. Glutaraldehyde: a new fixative in endodontics. J Dent Res 1973; 52(3):601 (abstract $n^{\circ} 48$ ).

35. Leach ED. A new synergized glutaraldehydephenate sterilizing solution and concentrated desinfectant. Infect Control 1981;2(1):26-30.

36. Tsai T-P, Su H-L, Tseng L-H. Glutaraldehyde preparations and pulpotomy in primary molars. Oral surg 1993;76(3):346-50.

37. Kopel HM, Bernick S, Zachrisson E, De Romero SA. The effects of glutaraldehyde on primary pulp tissues following coronal amputation: an in vivo histologic study. J Dent Child 1980;47(6):42530.

38. Fuks AB, Bimstein E, KLein H. Assesment of a $2 \%$ buffered glutaraldehyde solution in pulpotomized primary teeth of school children: a preliminary report. J Pedod 1986; 10(4):32330.

39. Fuks AB, Bimstein E, Guelmann M, Klein H. Assesment of a 2 percent buffered glutaraldehyde solution in pulpotomized primary teeth of school children. J Dent Child 1990;57(5):371-5.

40. Alacam A. Pulpal tissue changes following pulpotomies with formocresol, glutaraldehydecalcium hydroxide, glutaraldehyde-zinc oxide eugenol pastes in primary teeth. J Pedod 1989; 13(2):123-32.

41. Fernández H, Mendoza A, Solano E. Pulpotomía: estudio comparado entre glutaraldehido al $4 \%$ y formocresol al $20 \%$ en dientes temporales vitales. Odontol Pediatr 2000;8(1):19-25. 
42. Prakash C, Chandra S, Aiswal SN. Formocresol and glutaraldehyde pulpotomies in primary teeth. J Pedod 1989;13(4):314-22.

43. García-Godoy F. Clinical evaluation of glutaraldehyde pulpotomies in primary teeth. Acta Odontol Pediatr 1983;4(2):41-4.

44. Shumayrikh NM, Adenubi JO. Clinical evaluation of glutaraldehyde with calcium hydroxide and glutaraldehyde with zinc oxide eugenol in pulpotomy of primary molars. Endod Dent Traumatol 1999; 15(6):259-64.

45. García-Godoy F. A 42 month clinical evaluation of glutaraldehyde pulpotomies in primary teeth. J Pedod 1986;10(2):148-55.

46. Alacam A. Long term effects of primary teeth pulpotomies with formocresol, glutaraldehydecalcium hydroxide and glutaraldehyde-zinc oxide eugenol on succudaneous teeth. J Pedod 1989; 13(4):307-13.

47. Ibricevic $\mathrm{H}, \mathrm{Al}$-Jane $\mathrm{Q}$. Ferric sulfate as a pulpotomy agent in primary teeth: twenty month clinical follow-up. J Clin Pediatr Dent 2000;24(4): 269-72.

48. Casas MJ, Kenny DJ, Johnson DH, Judd PL. Long-term outcomes of primary molar ferric sulfate pulpotomy and root canal therapy. Pediatr Dent 2004;26(1):44-8.

49. Agamy HA, Baky NS, Mounir MMF, Avery DR. Comparision of mineral trioxide aggregate and formocresol as pulp-capping agentes in pulpotomized primary teeth. Pediatr Dent 2004; 26(4):302-9.

50. Maroto-Edo M, Barbería-Leache E, Planells P. Estudio clínico del agregado trióxido mineral en pulpotomías de molares temporales: estudio de 15 meses. RCOE 2004;9(1):23-30.

51. Maroto M, Barbería E, Planells P, García-Godoy $F$. Dentin bridge formation after mineral trioxide aggregate (MTA) pulpotomies in primary teeth. Am J Dent 2005;18(3):151-4.

52. Naik S, Hegde AM. Mineral trioxide aggregate as a pulpotomy agent in primary molars: an in vivo study. J Indian Soc Pedod Preven Dent 2005; 23(1):13-6.

53. Holan G, Eidelman E, Fuks AB. Long-term evaluation of pulpotomy in primary molars using mineral trioxide aggregate. Pediatr Dent 2005; 27(2):129-36.

54. Feigal RJ, Messer HH. A critical look at glutaraldehyde. Pediatr Dent 1990;12(2):69-71.

55. Ranly DM, Horn D, Hubbard GB. Assesment of the systemic distribution and toxicity of glutaraldehyde as a pulpotomy agent. Pediatr Dent 1989;11(1):8-12.

56. Myers DR, Pashley DH, Lake FT, Burnham D, Kalathoor S, Waters R. Systemic absortion of 14C-glutaraldehyde from glutaraldehyde-trated pulpotomy sites. Pediatr Dent 1986;8(2):134-8.

\section{CORRESPONDENCIA}

Dr. J. Calatayud

Departamento de Profilaxis, Odontopediatría y Ortodoncia

Facultad de Odontología

Universidad Complutense

Ciudad Universitaria.

28040 Madrid

E-mail: calata@odon.ucm.es 